\title{
Perfil Antropométrico del Canoísta Joven de Aguas Tranquilas
}

\author{
Anthropometric Profile of Young Male Sprint Canoeist
}

"Fernando Alacid; ***José María Muyor \& ${ }^{* * * *}$ Pedro Ángel López-Miñarro

ALACID, F.; MUYOR, J. M. \& LÓPEZ-MIÑARRO, P. A. Perfil antropométrico del canoísta joven de aguas tranquilas. Int. J. Morphol., 29(3):835-840, 2011.

RESUMEN: Los objetivos de este trabajo fueron describir las características antropométricas de hombres canoístas de categoría cadete y comparar su somatotipo y proporcionalidad con palistas olímpicos. Treinta y tres canoístas de 15 a 16 años de edad, fueron valorados usando una batería de 41 medidas antropométricas. Se calculó la composición corporal, el somatotipo y los valores $\mathrm{Z}$ utilizando la estrategia Phantom de proporcionalidad. El somatotipo medio de los canoístas obtuvo la clasificación de mesomorfo balanceado. De la comparación con los palistas de categoría olímpica, destacó la menor mesomorfia así como los valores inferiores de proporcionalidad en los perímetros y diámetros del tronco de las extremidades superiores de los canoístas cadetes. La información aportada en este estudio puede ser utilizada como guía en el proceso de detección de talentos en canoístas jóvenes de aguas tranquilas.

PALABRAS CLAVE: Antropometría; Somatotipo; Proporcionalidad; Composición corporal; Piragüismo/Canotaje.

\section{INTRODUCCIÓN}

La evaluación antropométrica es una de las valoraciones más usada para la detección de talentos en diferentes deportes (Reilly, 2008). De hecho, las dimensiones antropométricas, la forma, proporcionalidad y composición corporal de un deportista juegan un papel determinante en el potencial éxito en una determinada especialidad deportiva (Norton et al., 1996).

En el caso del piragüismo o canotaje de aguas tranquilas, tanto los kayakistas como canoístas de elite han sido analizados en varios estudios (Ackland et al., 2003; Aitken \& Jenkins, 1998; Cermak et al., 1975; Fry \& Morton, 1991; Misigoj-Durakovic \& Heimer, 1992; Shephard, 1987; van Someren \& Palmer, 2003). Los palistas de elite han sido descritos como poseedores de determinadas características no observadas en la población normal, como un mayor peso atribuido a una gran masa magra y baja adiposidad, grandes longitudes, diámetros y perímetros en el tronco y miembros superiores, así como una prevalencia de la mesomorfia y homogeneidad en la forma y el tamaño corporal (Ackland et al.). Además, algunos trabajos han encontrado correlaciones significativas de las dimensiones antropométricas de los kayakistas con el rendimiento en agua, en diferentes distancias. La talla sentado y el perímetro mesoesternal han sido correlacionados de forma significativa con el tiempo en $1000 \mathrm{~m}$ (Fry \& Morton); el perímetro mesoesternal y el diámetro biepicondíleo del húmero con el rendimiento en 500 m (van Someren \& Howatson, 2008); y los perímetros del brazo, brazo contraído, antebrazo y mesoesternal, junto con el diámetro biepicondíleo del húmero con el tiempo en $200 \mathrm{~m}$ (van Someren \& Palmer).

Sin embargo, la mayor parte de los estudios realizados han sido desarrollados sobre muestras compuestas principalmente por kayakistas. Entre los pocos trabajos publicados que aportan datos sobre canoístas, Misigoj-Durakovic \& Heimer compararon las características antropométricas de 18 kayakistas y 11 canoístas, candidatos a participar en la Universiada de 1987, concluyendo que no había diferencias significativas entre los palistas de ambas disciplinas. Basándose en esas similitudes, Ackland et al. midieron y analizaron las características antropométricas de 50 kayakistas y canoístas de aguas tranquilas que participaron en los JJ.OO. de Sydney 2000, aunque sólo el 12\% de la

\footnotetext{
* Departamento de Actividad Física y Deporte. Facultad de Ciencias del Deporte. Universidad de Murcia. España.

** Área de Expresión Corporal. Facultad de Ciencias de la Educación. Universidad de Almería. España.

**** Departamento de Expresión Plástica, Musical y Dinámica. Facultad de Educación. Universidad de Murcia. España

Este estudio ha sido financiado con una ayuda de la Fundación Séneca-Agencia de Ciencia y Tecnología de la Región de Murcia (II PCTRM 20072010) $c o n n^{\circ}$ 11951/PI/09 (Evolución de las curvaturas sagitales del raquis, extensibilidad isquiosural, dolor lumbar y características antropométricas de piragüistas de élite).
} 
muestra estaba compuesta por canoístas, y no se aportaron datos específicos de esta disciplina.

Por otro lado, son escasos los trabajos en los que se describan las características antropométricas de palistas jóvenes y se han centrando principalmente en las edades de 13 a 14 años de edad (Alacid et al., 2011; Carrasco et al., 2005; Cuesta et al., 1991). Por todo ello, los objetivos del presente trabajo fueron: 1) describir las características antropométricas, composición corporal, somatotipo y proporcionalidad de hombres canoístas de categoría cadete, con el fin de establecer un perfil antropométrico de referencia que pueda ser usado en el proceso de detección de talentos deportivos en dicha disciplina; y 2) comparar el somatotipo y la proporcionalidad con los datos de referencia en palistas de elite.

\section{MATERIAL Y MÉTODO}

Un total de 33 hombres canoístas de categoría cadete (edad: 15 - 16 años) participaron en este estudio. Todos ellos fueron seleccionados por encontrarse entre los mejores de su categoría, para su asistencia a las Concentraciones Nacionales de Cadetes en los años 2009 y 2010, realizadas dentro del Programa Nacional de Tecnificación del Consejo Superior de Deportes y la Real Federación Española de Piragüismo.

Los padres y los deportistas fueron informados de los objetivos y métodos del estudio y se obtuvo un consentimiento informado de sus tutores.

Las variables que componen el perfil completo descrito por la ISAK (International Society for the Advancement of Kinanthropometry), más la envergadura y el diámetro biestiloideo fueron tomadas por un antropometrista acreditado de nivel II siguiendo las indicaciones descritas por dicha organización (Marfell-Jones et al., 2006). Las medidas se tomaron dos o tres veces, si la diferencia entre las dos primeras era superior al 5\% en pliegues y al $1 \%$ en el resto de medidas, tomando la media o la mediana, respectivamente, para realizar los análisis posteriores.

Para la determinación del peso se utilizó una báscula SECA 862 (SECA, Alemania) de $100 \mathrm{~g}$ de precisión; para los pliegues un plicómetro Harpenden (British Indicators, UK) de $0,2 \mathrm{~mm}$ de precisión; para la envergadura y los perímetros una cinta métrica inextensible milimetrada Lufkin W606PM (Lufkin, EE.UU.); y para la talla, talla sentado, alturas, longitudes y diámetros, un antropómetro Siber-Hegner GPM (Suiza), ambos instrumentos con una precisión de $0,1 \mathrm{~cm}$.
Para la determinación de la composición corporal se utilizó la estrategia de cinco componentes de Kerr (1988). La comparación con palistas de elite se realizó a través del análisis del somatotipo según la metodología descrita por Carter \& Heath (1990) y del cálculo de los valores Z según la estrategia del Phantom de Ross \& Marfell-Jones (1991).

\section{RESULTADOS Y DISCUSIÓN}

La Tabla I muestra las características antropométricas de los canoístas de categoría cadete analizados. En lo que se refiere a la talla y el peso, la muestra se sitúa entre el percentil 50 y 75 en ambas variables (171,6 y $176,0 \mathrm{~cm}$ para la talla; 62,2 y $70,2 \mathrm{~kg}$ para el peso, respectivamente). Al compararlo con las tablas de crecimiento de la población española (Carrascosa et al., 2008), esta localización no indica que los canoístas jóvenes sean más altos o pesados que la población normal, tal y como ocurre con los palistas de elite (Ackland et al.; Cermak et al.; Tesch \& Lindeberg, 1984). Por otro lado, estos resultados suponen un incremento en más de 12 $\mathrm{cm}$ para la talla y casi $13 \mathrm{~kg}$ en el peso respecto a canoístas de 13 y 14 años de edad (Carrasco et al.).

El somatotipo medio y la composición corporal de los canoístas cadetes se muestran en la Tabla II. Respecto al somatotipo, los canoístas son descritos como mesomorfos balanceados, coincidiendo con el resultado obtenido por palistas de elite (1,6-5,7-2,2) (Ackland et al.), aunque con valores bastante inferiores de mesomorfia y superiores de endomorfia propios de una población que aún no ha finalizado los procesos de crecimiento y que se encuentra en los inicios de su carrera deportiva (Fig. 1). La dispersión morfogénica media del somatotipo (somatotype attitudinal mean), como una medida de la dispersión media de los somatotipos individuales respecto al somatotipo medio, fue de 1,15 puntos, indicando una gran homogeneidad en estos palistas, coincidiendo con el valor alcanzado por los palistas participantes en los JJ.OO. de Sydney $(1,1)$ (Ackland et al.).

Los valores obtenidos en la composición corporal de los canoístas cadetes fueron del $12,1 \%$ en la masa ósea y residual, el $45,7 \%$ en la masa muscular y el $24,5 \%$ en la masa grasa. Son pocos los trabajos que utilizan este método de fraccionamiento de las masas corporales, y los existentes ha sido realizados con deportistas de elite de otras especialidades cuya comparación con los jóvenes canoístas reporta datos inferiores en masa grasa y superiores en masa muscular propios de un mayor desarrollo físico y deportivo (Almagià et al., 2009; Holway \& Garavaglia, 2009). Por otro lado, aunque en este modelo penta-compartimental no se utiliza el peso total en el cálculo de ninguna de las masas, el error medio respecto a la 
Tabla I. Características antropométricas de los canoístas de categoría cadete.

\begin{tabular}{|c|c|c|c|c|}
\hline Variable & Media & $\mathrm{DE}$ & Máximo & Mínimo \\
\hline Edad (años) & 15,6 & 0,6 & 16,5 & 14,5 \\
\hline Peso (kg) & 68,6 & 7,1 & 85,1 & 56,8 \\
\hline Talla $(\mathrm{cm})$ & 172,9 & 5,3 & 187,9 & 164,8 \\
\hline Talla sentado $(\mathrm{cm})$ & 90,8 & 3,0 & 96,8 & 86,6 \\
\hline Envergadura (cm) & 176,4 & 6,3 & 193,0 & 164,0 \\
\hline Sumatorio de 6 pliegues (mm) & 59,2 & 15,7 & 98,0 & 32,2 \\
\hline Sumatorio de 8 pliegues (mm) & 74,7 & 20,0 & 123,9 & 41,6 \\
\hline PR Cabeza (cm) & 55,6 & 1,4 & 58,5 & 52,7 \\
\hline PR Cuello (cm) & 34,6 & 1,6 & 38,9 & 32,2 \\
\hline PR Brazo relajado $(\mathrm{cm})$ & 28,5 & 2,1 & 32,2 & 25,0 \\
\hline PR Brazo contraído (cm) & 31,5 & 2,2 & 36,0 & 28,1 \\
\hline PR Antebrazo (cm) & 26,1 & 1,3 & 28,5 & 24,0 \\
\hline PR Muñeca $(\mathrm{cm})$ & 16,1 & 0,7 & 18,0 & 14,9 \\
\hline PR Mesoesternal (cm) & 94,6 & 4,2 & 105,0 & 87,8 \\
\hline PR Cintura (cm) & 75,6 & 3,9 & 89,0 & 70,0 \\
\hline PR Cadera (cm) & 92,9 & 4,1 & 104,4 & 85,5 \\
\hline PR Muslo (1 cm línea glutea) $(\mathrm{cm})$ & 54,2 & 3,2 & 60,2 & 48,9 \\
\hline PR Muslo medio (cm) & 50,7 & 2,9 & 56,7 & 44,9 \\
\hline PR Pierna (máx.) (cm) & 35,4 & 2,5 & 40,5 & 27,7 \\
\hline PR Tobillo (cm) & 22,5 & 1,3 & 25,8 & 20,4 \\
\hline L Brazo (cm) & 32,5 & 1,6 & 36,6 & 29,3 \\
\hline L Antebrazo (cm) & 25,7 & 1,2 & 29,8 & 24,0 \\
\hline L Mano $(\mathrm{cm})$ & 19,2 & 0,8 & 21,1 & 17,9 \\
\hline L Muslo (cm) & 43,5 & 2,5 & 49,4 & 38,5 \\
\hline L Pierna (medial) $(\mathrm{cm})$ & 38,5 & 1,9 & 43,6 & 35,4 \\
\hline L Pie $(\mathrm{cm})$ & 25,9 & 1,2 & 28,2 & 24,3 \\
\hline H Ileoespinal (cm) & 95,7 & 4,4 & 106,3 & 88,7 \\
\hline H Trocantérea (cm) & 90,3 & 4,6 & 102,5 & 83,3 \\
\hline H Tibial lateral $(\mathrm{cm})$ & 46,3 & 2,8 & 55,5 & 40,6 \\
\hline D Biacromial (cm) & 39,4 & 1,6 & 42,2 & 36,1 \\
\hline D Biileocrestal (cm) & 27,8 & 1,3 & 31,2 & 25,7 \\
\hline D Trasnverso del tórax (cm) & 29,8 & 1,6 & 34,0 & 26,6 \\
\hline D Anteroposterior del tórax (cm) & 19,4 & 1,1 & 21,6 & 17,2 \\
\hline D Biepicondíleo del húmero (cm) & 6,9 & 0,4 & 8,0 & 6,2 \\
\hline D Bicondíleo del fémur (cm) & 9,7 & 0,5 & 10,5 & 8,4 \\
\hline D Biestiloideo $(\mathrm{cm})$ & 5,6 & 0,3 & 6,4 & 4,8 \\
\hline
\end{tabular}

DE: desviación estándar. PR: perímetro. L: longitud. H: altura. D: diámetro.

suma de las masas individuales derivadas fue de 1,8 $\pm 1,6 \mathrm{~kg}$, aspecto que refuerza el uso de esta metodología para la determinación de la composición corporal.

Las características en cuanto a proporcionalidad de los canoístas de categoría cadete comparadas con los palistas de elite mostraron una estructura general con bastantes similitudes (Fig. 2). Las principales diferencias respecto a los palistas de elite se centraron en unas proporciones claramente inferiores en los perímetros del brazo contraído, mesoesternal y de la cintura, seguido de inferiores diámetros biacromial, anteroposterior del tórax y biepicondíleo 
Tabla II. Somatotipo y composición corporal de las canoístas cadetes.

\begin{tabular}{lcccc}
\hline Variable & Media & DE & Máximo & Mínimo \\
\hline Endomorfia & 2,4 & 0,7 & 4,0 & 1,2 \\
Mesomorfia & 4,9 & 0,8 & 6,4 & 3,1 \\
Ectomorfia & 2,4 & 0,8 & 3,9 & 0,7 \\
Masa de la piel (\%) & 5,6 & 0,4 & 6,4 & 4,9 \\
Masa ósea (\%) & 12,1 & 1,0 & 14,5 & 10,1 \\
Masa grasa (\%) & 24,5 & 2,6 & 29,1 & 19,4 \\
Masa muscular (\%) & 45,7 & 2,3 & 50,1 & 41,1 \\
Masa residual (\%) & 12,1 & 0,7 & 13,5 & 10,3 \\
Masa de la piel (kg) & 3,8 & 0,2 & 4,4 & 3,4 \\
Masa ósea (kg) & 8,2 & 0,8 & 10,1 & 6,9 \\
Masa grasa (kg) & 16,6 & 3,2 & 25,1 & 11,6 \\
Masa muscular (kg) & 30,9 & 4,1 & 40,1 & 23,6 \\
Masa residual (kg) & 8,1 & 0,9 & 11,0 & 6,8 \\
Diferencia respecto al peso real (kg) & 1,8 & 1,6 & 5,3 & 0,0 \\
\hline
\end{tabular}

DE: desviación estándar.

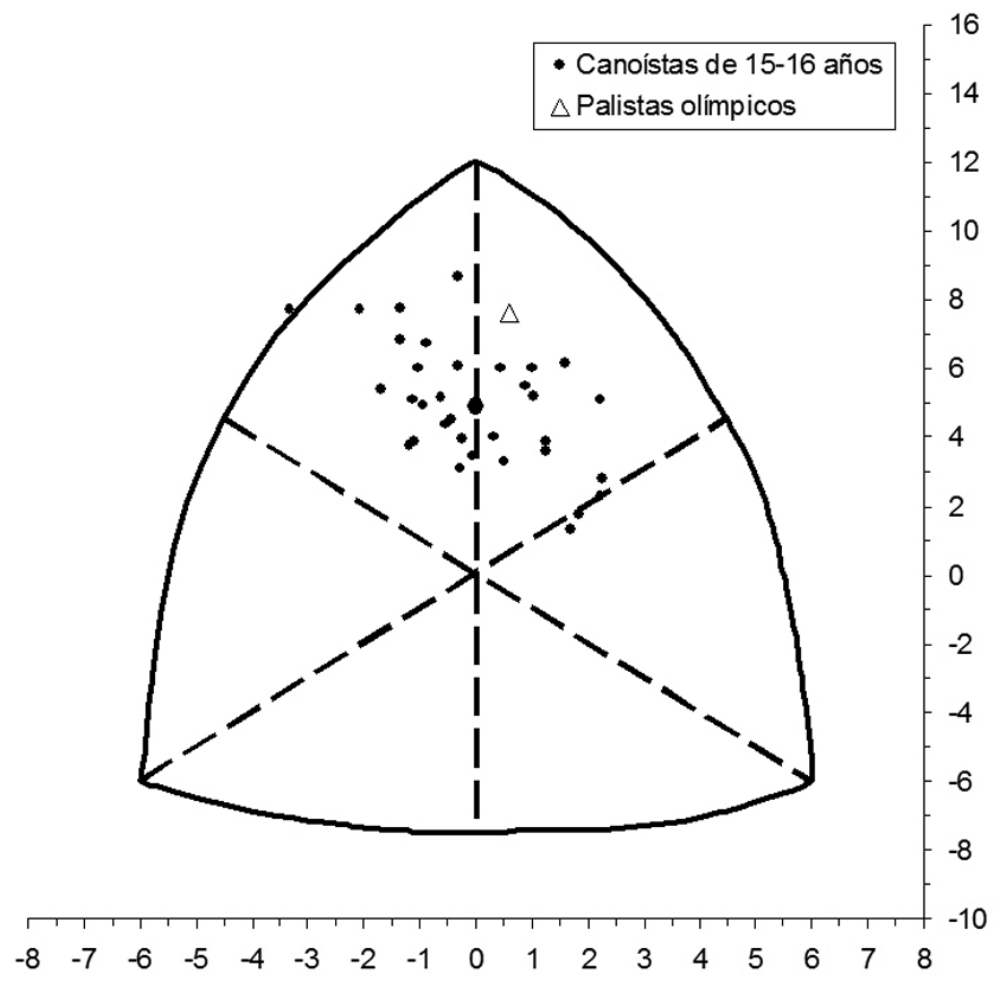

Fig. 1. Somatopuntos de los canoístas de 15 y 16 años y de los palistas olímpicos (Ackland et al., 2003). El círculo grande representa el valor medio.

del húmero y una menor adiposidad, características que definen al palista de elite de aguas tranquilas (Ackland et al.). Sin embargo, los canoístas presentaron un proporcionalmente mayor bicondíleo del fémur, algo que podría tener relación con una mayor implicación de los miembros inferiores en esta disciplina.
Como conclusión, este trabajo aporta una importante cantidad de información normativa sobre las características antropométricas del hombre canoísta de categoría cadete que puede utilizarse de referencia en el proceso de valoración y entrenamiento de canoístas jóvenes, debido fundamentalmente al nivel deportivo de la muestra analizada. Los canoístas presentaron un somatotipo clasificado como mesomorfo balanceado al igual que los palistas de elite, pero con valores bastante inferiores de mesomorfia; mientras que las principales diferencias en el análisis de la proporcionalidad se centraron en los perímetros y diámetros del tronco y los miembros superiores. La información aportada en este estudio puede ser utilizada como guía en el proceso de detección de talentos en canoístas jóvenes de aguas tranquilas.

\section{AGRADECIMIENTOS}

Los autores agradecen su colaboración a la Real Federación Española de Piragüismo, así como a los entrenadores y deportistas que participaron en el estudio. 


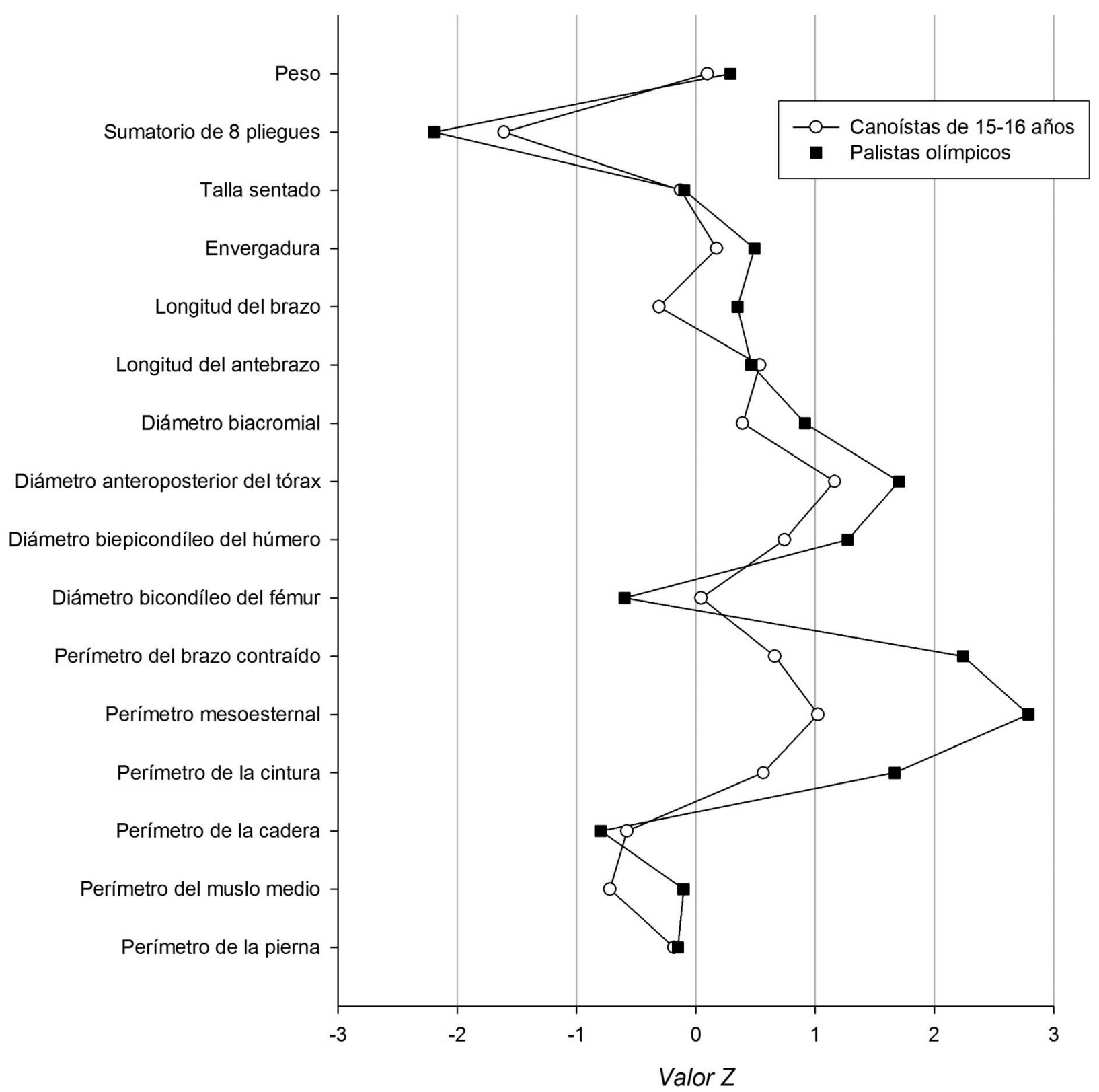

Fig. 2. Proporcionalidad (valores Z) de los canoístas de 15-16 años comparada con palistas olímpicos (Ackland et al., 2003).

ALACID, F.; MUYOR, J. M. \& LÓPEZ-MIÑNARO, P. A. Anthropometric profile of young male sprint canoeist. Int. J. Morphol., 29(3):835-840, 2011.

SUMMARY: The aim of this study was to describe kinanthropometric characteristics of young male sprint canoeists and to compare their somatotype and proportionality with Olympic paddlers. Thirty three young sprint canoeists, aged 15 and 16 years old, were assessed using a battery of 41 anthropometric dimensions. Body composition, somatotypes and Phantom Z-scores were calculated. Mean somatotype of male canoeists was best described as balanced mesomorphy. Young canoeists had lower ratings of mesomorphy and lower proportional dimensions in upper body girths and breadths than Olympic paddlers. The data provided in this study could be used as a guideline for talent identification in sprint canoeing.

KEY WORDS: Anthropometry; Somatotype; Proportionality; Body composition; Canoeing. 


\section{REFERENCIAS BIBLIOGRÁFICAS}

Ackland, T. R.; Ong, K. B.; Kerr, D. A. \& Ridge, B. Morphological characteristics of Olympic sprint canoe and kayak paddlers. J. Sci. Med. Sport, 6(3):285-94, 2003.

Aitken, D. A. \& Jenkins, D. G. Anthropometric-based selection and sprint kayak training in children. J. Sports Sci., 16(6):539-43, 1998.

Alacid, F.; Lopez-Minarro, P. A.; Martínez, I. \& Ferrer-López, V.Anthropometric indexes in young paddlers. Rev. Int. Med. Cienc. Act. Fís. Deporte, 10(41):58-76, 2011.

Almagià, A.; Rodriguez, F.; Barranza, F. O.; Linaza, P. J.; Ivanovic, D. \& Binvignat, O. Perfil antropométrico de jugadores profesionales de voleibol sudamericano. Int. J. Morphol., 27(1):53-7, 2009.

Carrasco, L.; Martínez, E. \& Nadal, C. Perfil antropométrico, somatotipo y composición corporal de jóvenes piragüistas. Rev. Int. Med. Cienc. Act. Fís. Deporte, 5(19):270-82, 2005.

Carrascosa, A.; Fernández, J. M.; Fernández, C.; Ferrández, A.; López-Siguero, J. P.; Sánchez, E.; Sobradillo, B. \& Yeste, D. Spanish cross-sectional growth study 2008. Part II. Height, weight and body mass index values from birth to adulthood. An. Pediatr. (Barc.), 68(6):552-69, 2008.

Carter, J. E. L. \& Heath, B. H. Somatotyping: development and application. Cambridge, Cambridge University Press, 1990.

Cermak, J.; Kuta, I. \& Parizkova, J. Some predispositions for top performance in speed canoeing and their changes during the whole year training program. J. Sports Med. Phys. Fitness, 15(3):243-51, 1975.

Cuesta, G.; Polo, J. M. \& Padilla, S. Correlación entre la marca deportiva obtenida en test de campo y parámetros fisiológicos obtenidos en laboratorio, en piragüistas adolescentes. Apunts, 18:130-42, 1991.

Fry, R. W. \& Morton, A. R. Physiological and kinanthropometric attributes of elite flatwater kayakists. Med. Sci. Sports Exerc., 23(11):1297-301, 1991.

Holway, F. E. \& Garavaglia, R. Kinanthropometry of Group I rugby players in Buenos Aires, Argentina. J. Sports Sci., 27(11):1211-20, 2009.

Kerr, D. A. An anthropometric method for fractionation of skin, adipose, bone, muscle and residual masses in males and females age 6 to 77 years. M.Cs. Kinesiology Tesis, Simon Fraser University, British Columbia, 1988.
Marfell-Jones, M.; Olds, T.; Stewart, A. \& Carter, L. International standards for anthropometric assessment. Potchefstroom (South Africa), ISAK, 2006.

Misigoj-Durakovic, M. \& Heimer, S. Characteristics of the morphological and functional status of kayakers and canoeists. J. Sports Med. Phys. Fitness, 32(1):45-50, 1992.

Norton, K.; Olds, T.; Olive, S. \& Craig, N. Anthropometry and Sports Performance. En: Norton, K. \& Olds, T. (Eds.). Anthropometrica. Sydney, University of New South Wales Press, 1996. pp.287-364.

Reilly, T. The international face of sports science through the window of the Journal of Sports Sciences - with a special reference to kinanthropometry. J. Sports Sci, 26(4):349-63, 2008.

Ross, W. D. \& Marfell-Jones, M. Kinanthropometry. En J. MacDougal, H. Wenger \& H. Green (Eds.), Physiological testing of the high performance athlete. $2^{\text {nd }} \mathrm{ed}$. Champaign (IL), Human Kinetics, 1991. pp.223-308.

Shephard, R. J. Science and medicine of canoeing and kayaking. Sports Med., 4(1):19-33, 1987.

Tesch, P. A. \& Lindeberg, S. Blood lactate accumulation during arm exercise in world class kayak paddlers and strength trained athletes. Eur. J. Appl. Physiol. Occup. Physiol., 52(4):441-5, 1984.

van Someren, K. A. \& Howatson, G. Prediction of flatwater kayaking performance. Int. J. Sports Physiol. Perform., 3(2):207-18, 2008.

van Someren, K. A. \& Palmer, G. S. Prediction of 200-m sprint kayaking performance. Can. J. Appl. Physiol., 28(4):505$17,2003$.

Dirección para correspondencia:

Fernando Alacid Cárceles

Facultad de Ciencias del Deporte

Universidad de Murcia.

C/Argentina, s/n.

30720. Santiago de la Ribera

Murcia - ESPAÑA

Tel. (+34) 868888653

Email: fernando.alacid@um.es
Recibido : 18-01-2011

Aceptado: 19-04-2011 